\title{
Intracranial pressure and cerebral blood flow velocity in preterm infants with posthaemorrhagic ventricular dilatation
}

\author{
W J Maertzdorf, J S H Vles, E Beuls, A L M Mulder, C E Blanco
}

Arch Dis Child Fetal Neonatal Ed 2002;87:F185-F188

See end of article for authors' affiliations

Correspondence to

Dr Maertzdorf, Department

of Paediatrics, Academic

Hospital Maastricht, PO

5800, $6202 \mathrm{AZ}$

Maastricht, The

Netherlands;

wma@skin.azm.nl

Accepted 14 May 2002

\begin{abstract}
Aim: To determine the volume of cerebrospinal fluid (CSF) that should be tapped in preterm infants with posthaemorrhagic ventricular dilatation as guided by intracranial pressure (ICP) and cerebral blood flow velocity (CBFV).

Methods: The total number of measurements was 106 in 22 infants. Birth weights ranged from 630 to $2050 \mathrm{~g}$, gestational age from 24.5 to 30.3 weeks, and age at insertion from 12 to 67 days. A subcutaneous ventricular catheter reservoir for repetitive CSF drainage was placed when the diameter of a ventricle was $>4 \mathrm{~mm}$ above the 97th centile. A volume of $5 \mathrm{ml} / \mathrm{kg}$ body weight was removed twice daily. ICP and CBFV were determined before and after CSF tapping.

Results: If the ICP after tapping exceeded $7 \mathrm{~cm} \mathrm{H}_{2} \mathrm{O}$, tapping did not result in a significant improvement in CBFV. If the ICP before tapping was less than $6 \mathrm{~cm} \mathrm{H}_{2} \mathrm{O}$, tapping also had no effect on CBFV. Longitudinal studies in individual infants showed a slight correlation between ICP and CBFV.

Conclusion: Volume of repetitive CSF drainage in preterm infants with posthaemorrhagic ventricular dilatation guided by ICP and CBFV may be a useful technique. An ICP of about $6 \mathrm{~cm} \mathrm{H}_{2} \mathrm{O}$ is the cut off point for CSF drainage.
\end{abstract}

$\mathrm{P}$ osthaemorrhagic ventricular dilatation (PHVD) is a major complication of intraventricular haemorrhages in very preterm infants. ${ }^{12}$ It may lead to an impaired neurological prognosis, caused by a raised intracranial pressure (ICP) and concomitant decreased cerebral blood perfusion. ${ }^{34}$ Therefore, early intervention to decrease the ICP may improve the long term outcome. ${ }^{5}$

Different treatment modalities have been studied, ${ }^{6-8}$ of which repetitive removal of cerebrospinal fluid (CSF) seems to be the only effective one. Repetitive lumbar liquid punctures are difficult to perform and not always possible in very small infants. Insertion of a subcutaneous reservoir for repetitive CSF removal to bridge the period until placement of continuous ventriculoperitoneal drainage, or even to avoid placement of a permanent drainage system, is gaining popularity. ${ }^{9}$ However, the frequency and volume of CSF removal are not well defined. As high ICP can influence cerebral perfusion, the study of cerebral blood flow velocity ( $\mathrm{CBFV}$ ) may provide some guidance. ${ }^{5}$

The aim of this study was to investigate the relation between CBFV and ICP in preterm infants with PHVD and to assess a subcutaneous access device for intermittent CSF drainage in order to determine frequency and volume of CSF removal.

\section{PATIENTS AND METHODS}

After parental consent, 22 preterm infants ( 10 boys, 12 girls) were included in the study. Table 1 gives gestational age, birth weight, and age at the start of CSF removal.

Using an ATL-Ultramark machine with a 5.0, 7.5, and 10 $\mathrm{MHz}$ transducer, we measured the width of each lateral ventricle in the coronal view just posterior to the interventricular foramina three times a week. We used the criterion of Levene $^{10}$ - that is, a ventricular diameter $>4 \mathrm{~mm}$ above the 97th centile for CSF removal. Intervention was started as soon as the criterion was achieved.

A subcutaneous ventricular catheter reservoir (type Ommaya) was inserted, and for access the catheter was positioned
Table 1 Characteristics of the infants studied $(n=22)$

\begin{tabular}{lll}
\hline & Median & Range \\
\hline Gestational age (weeks) & 27.1 & $24.5-30.3$ \\
Birth weight (g) & 940 & $630-2050$ \\
Age at insertion of reservoir (days) & 20 & $12-67$ \\
Number of aspirations per infant & 5 & $2-8$ \\
\hline
\end{tabular}

in the frontal horn of the right ventricle. The procedure was performed under general anaesthesia under aseptic conditions in an open incubator in the intensive care unit or in the operation theatre. Antibiotic prophylaxis (rifampycin + vancomycin) was given for 24 hours. Leakage of CSF during the insertion procedure was avoided and ICP was not measured during the surgical procedure.

After 24 hours, $5 \mathrm{ml} \mathrm{CSF} / \mathrm{kg}$ body weight was removed twice a day. Punctures were performed under aseptic conditions using a 25 gauge butterfly needle. Using a T connector to a calibrated open ascending system, the ICP was measured before and after CSF removal. All punctures were performed when the infant was asleep or peacefully awake.

CBFV was measured using a continuous wave bidirectional Doppler velocimeter (Medasonic 10; Medasonics, Mountain View, California, USA). Flow velocity was measured before and at 15-20 minutes after CSF removal. Recordings from the anterior cerebral arteries were made through the open fontanel and from the mid cerebral arteries through the coronal suture. A $5 \mathrm{MHz}$ pencil probe was used, and Doppler frequency

Abbreviations: PHVD, posthaemorrhagic ventricular dilatation; ICP, intracranial pressure; CSF, cerebrospinal fluid; CBFV, cerebral blood flow velocity; EDFV, end diastolic flow velocity; MFV, mean flow velocity; RI, resistance index 

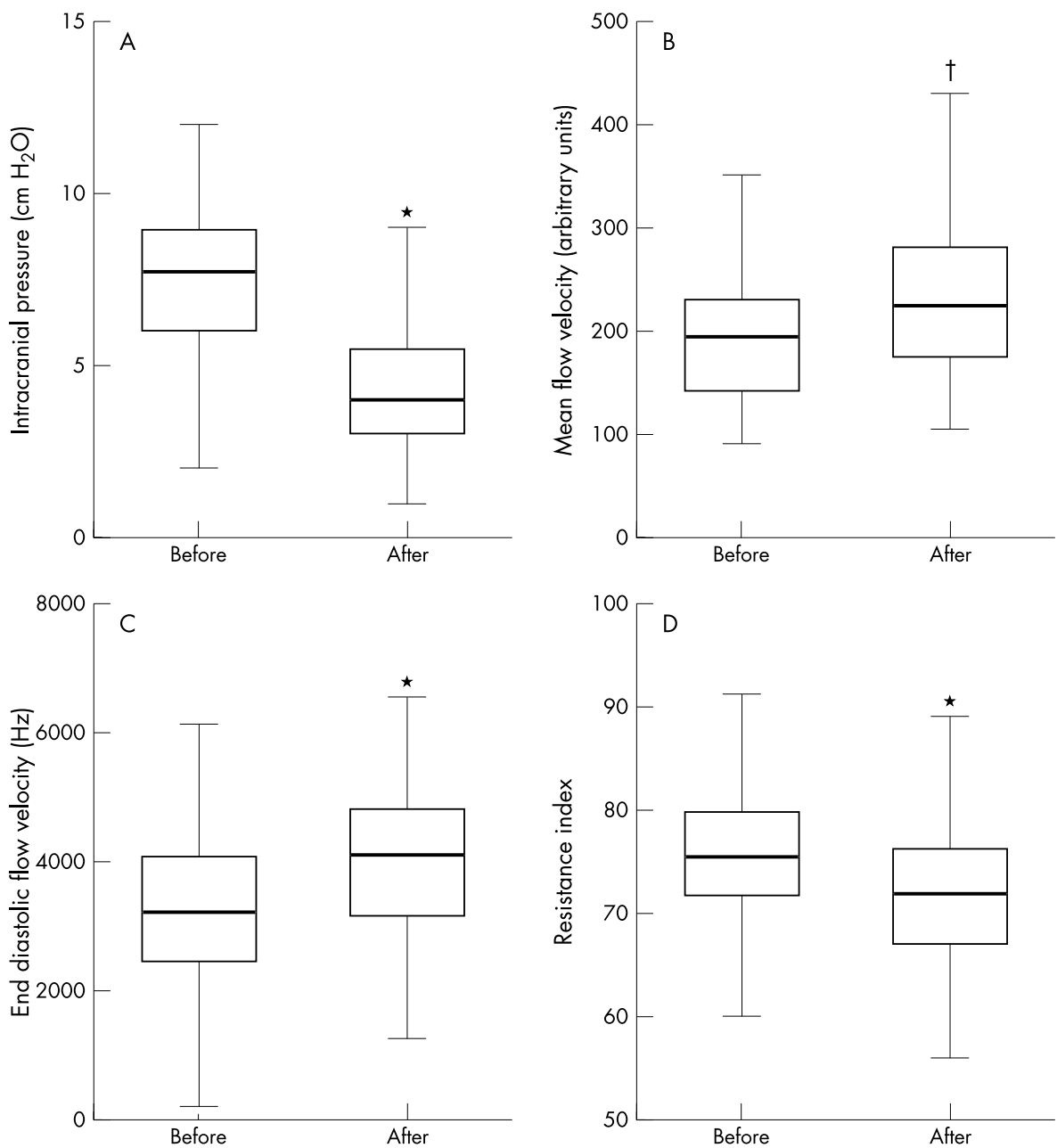

Figure 1 Changes in intracranial pressure (ICP) and cerebral blood flow variables after removal of cerebrospinal fluid on 106 occasions in 22 preterm infants. Box plots represent the median, interquartile values, and the range. (A) ICP; (B) mean flow velocity; (C) end diastolic flow velocity; (D) Resistance index. ${ }^{*} \mathrm{p}<0.01 ; \mathrm{\dagger p}<0.05$.

shifts were assessed by the zero crossing technique and expressed in Hz. Doppler signals were optimised through auditory and visual judgment by manipulating the angle of insonation, and velocity waveforms were recorded on a strip chart recorder. Recordings were defined as stable when the beat to beat coefficient of variation was less than $10 \%$. Eight consecutive waveforms were analysed to obtain peak systolic flow velocity (PSFV), end diastolic flow velocity (EDFV), mean flow velocity (MFV expressed as area under the curve per minute), and the resistance index (RI) as defined by Pourcelot $^{11}:$ RI $=($ PSFV-EDFV $) /$ PSFV.

For statistical analysis, the Wilcoxon signed ranks test was used to test the relation between ICP and different CBFV variables before and after CSF removal. The box plots represent the median, the $25 \%$, the $75 \%$, and the total range. Longitudinally obtained data on ICP and flow velocity variables in the individual infants were compared by analysis of variance. $p \leqslant 0.05$ was considered significant.

\section{RESULTS}

The number of ICP and CBFV measurements varied from two to eight for each patient. The total number of measurements was 106 in the 22 patients.

Heart rate and blood pressure were within the normal range for gestational age and did not change significantly after CSF removal.

No differences were found in changes in blood flow velocity between the anterior and mid cerebral arterial system. There- fore, all data presented in this study are from the anterior arterial system.

The mean ICP before the initial CSF tap was $10.5 \mathrm{~cm} \mathrm{H}_{2} \mathrm{O}$ (range 6.0-20.0). In every case, the ICP decreased significantly after CSF removal (fig l).

The decrease in ICP after CSF removal was accompanied by a concomitant increase in MFV $(p<0.05)$ and EDFV $(p<0.01)$. As the change in peak systolic flow velocity after CSF removal was not significant, the decrease in RI ( $p<0.01)$ can be mainly attributed to the increase in EDFV.

Using cross sectionally obtained data, no correlation was found between ICP and CBFV values.

In eight of the 22 infants, ICP and CBFV measurements were studied longitudinally on six or more occasions. Figure 2 presents an example of longitudinal measurements of ICP and RI in one of those infants. It shows that the RI decreases significantly if the ICP before tapping was high and decreased to normal levels after CSF removal. There was a slight tendency towards a correlation between ICP and CBFV in each of these eight infants, but because of the high interindividual variability, the number of infants studied was too low to draw conclusions.

Table 2 shows in detail the levels of significance of the changes in flow variables in relation to the decreases in ICP after CSF removal. These data show that an improvement in CBFV after CSF removal depends on the preceding ICP as well on the resulting fall in ICP. If the ICP at the start of the procedure was $<6.0 \mathrm{~cm} \mathrm{H} \mathrm{H}_{2} \mathrm{O}, \mathrm{CSF}$ removal did not result in a significant improvement in CBFV (21 occasions). In all 67 
Table 2 Levels of significance of changes in cerebral blood flow velocity after removal of cerebrospinal fluid

\begin{tabular}{|c|c|c|c|c|c|}
\hline \multirow[b]{2}{*}{ No of procedures } & \multicolumn{2}{|l|}{ ICP $\left(\mathrm{cm} \mathrm{H}_{2} \mathrm{O}\right)$} & \multicolumn{3}{|c|}{$\mathrm{p}$ Values } \\
\hline & Before tapping & After tapping & MFV & EDFV & RI \\
\hline 3 & $\geqslant 10$ & $9-10$ & 0.144 & 0.068 & 0.068 \\
\hline 7 & & 7-9 & 0.699 & 0.053 & 0.048 \\
\hline 10 & & $<7$ & 0.139 & 0.037 & 0.047 \\
\hline 4 & 9 & $7-9$ & 0.180 & 0.180 & 0.655 \\
\hline 9 & & $<7$ & 0.011 & 0.008 & 0.008 \\
\hline 4 & 8 & $7-8$ & 0.593 & 0.102 & 0.593 \\
\hline 17 & & $<7$ & 0.001 & 0.003 & 0.022 \\
\hline 10 & 7 & $4-6$ & 0.017 & 0.008 & 0.092 \\
\hline 9 & & $<4$ & 0.001 & 0.001 & 0.004 \\
\hline 8 & 6 & $4-5$ & 0.069 & 0.161 & 0.161 \\
\hline 4 & & $<4$ & 0.028 & 0.047 & 0.139 \\
\hline 7 & 5 & $<4$ & 0.180 & 0.180 & 0.109 \\
\hline 14 & $\leqslant 4$ & $\leqslant 3$ & 0.073 & 0.152 & 0.093 \\
\hline
\end{tabular}

ICP, Intracranial pressure; EDFV, end diastolic flow velocity; MFV, mean flow velocity; RI, resistance index.

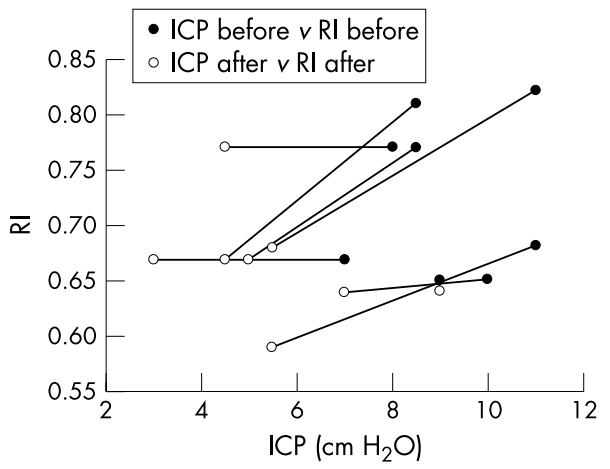

Figure 2 Example of decreases in intracranial pressure (ICP) and concomitant changes in resistance index (RI) after removal of cerebrospinal fluid eight consecutive times in the same infant.

occasions when the preceding ICP was $\geqslant 6.0 \mathrm{~cm} \mathrm{H}_{2} \mathrm{O}$ and fell to levels below $6.0 \mathrm{~cm} \mathrm{H}_{2} \mathrm{O}$ after CSF tapping, the CBFV increased significantly. If the ICP after CSF removal did not decrease to levels below $6.0 \mathrm{~cm} \mathrm{H}_{2} \mathrm{O}$ (18 occasions), CSF removal did not result in a significant improvement in CBFV. From these data, it may be concluded that, in very preterm infants, an improvement in CBFV can only be expected if CSF removal results in a decrease in ICP to levels of $6.0 \mathrm{~cm} \mathrm{H}_{2} \mathrm{O}$ or lower.

In two patients, the ICP stabilised after CSF tapping for periods of two and three weeks, and it was not necessary to continue with CSF removal or to proceed to permanent CSF drainage. In these two infants, CBFV improved significantly from baseline.

\section{DISCUSSION}

This study shows that intermittent CSF drainage in very preterm infants with PHVD is an effective way to treat increased ICP and its negative effect on cerebral blood perfusion. The cut off point for CSF drainage is about $6.0 \mathrm{~cm} \mathrm{H}_{2} \mathrm{O}$ ICP, as drainage below that level no longer results in improvement in perfusion. When studied longitudinally in the same infant, ICP shows a slight but non-significant correlation with CBFV. In preterm infants with $\mathrm{PHVD}, \mathrm{CBFV}$ measurements using the transcranial Doppler ultrasound technique may be a useful guideline for the time, volume, and frequency of CSF drainage.

The optimal method of treating PHVD has not yet been established. However, it is generally agreed that maintenance of blood perfusion by keeping the ICP within normal ranges is the primary goal to prevent neurological sequelae. ${ }^{12}$ All methods used to decrease ICP may have their disadvantages. Protracted repetitive lumbar CSF tapping is difficult to perform and undesirable in infants who need minimal handling. Moreover, it has no detectable benefits on ultimate developmental outcome. ${ }^{7}$ Placement of a permanent ventriculoperitoneal drain in these tiny infants is difficult to perform and often ineffective because of the high protein content of the CSF. Sustained external ventricular drains have the disadvantage of a high risk of infection. ${ }^{6}$ The use of recombinant tissue plasminogen activator needs further evaluation. ${ }^{13}$

A ventriculostomy reservoir for repetitive CSF tapping is one method used to temporarily relieve increased ICP until the placement of a permanent ventriculoperitoneal shunt device. In the hands of an experienced neurosurgeon, the procedure is easy to perform. A main advantage is that it can be executed on the ward for infants still in need of intensive care. ${ }^{14}$ Repetitive tapping gives minimal discomfort to the baby and may result in temporisation of the ventricular dilatation or may stabilise the process until definitive ventriculoperitoneal drainage. To prevent swings in ICP and therefore in cerebral perfusion, it is necessary to tap at regular intervals. The possibility of infection caused by frequent tapping necessitates strict aseptic handling.

Mean cerebral blood flow is mainly determined from the diastolic flow. As ICP rises, the arterial flow is more affected during diastole than during systole, resulting in an increase in RI. ${ }^{15-17}$ It is doubtful whether the RI can be used as an indicator for the timing of intervention, because it can vary widely between individual preterm infants. Hanlo et al ${ }^{18}$ found that cerebral blood flow variables do not correlate well with concomitantly measured values for ICP. Our study confirmed that flow velocities varied considerably between individual infants, and no correlation was found between the ICP and absolute flow velocities. However, in individual infants, we found a tendency towards a correlation between ICP and flow variables when studied longitudinally.

Cerebral blood flow and tissue perfusion are directly dependent on mean arterial blood pressure and ICP. ICP may 
be a good predictor of the need for CSF removal. However, ICP values for healthy preterm infants differ widely depending on the method used to measure it, and, moreover, wide ranges (5.6-10.1 $\mathrm{cm} \mathrm{H}_{2} \mathrm{O}$ ) have been reported in different studies using the same method. ${ }^{19}$ Mean ICP values in preterm infants with PHVD as reported in different studies vary widely from 12.2 to $17.7 \mathrm{~cm} \mathrm{H}_{2} \mathrm{O}$ when measured directly by the lumbar route. ${ }^{2021}$ These values cannot be compared, however, as the time of measurement after the onset of bleeding and the occurrence of PHVD are not reported. A Whitelaw (personal communication) found a mean ICP of $12.0 \mathrm{~cm} \mathrm{H}_{2} \mathrm{O}$ in preterm infants with PHVD, which agrees quite well with our finding of $10.5 \mathrm{~cm} \mathrm{H}_{2} \mathrm{O}$. In our study, CSF tapping was started when the ventricular size was $>4 \mathrm{~mm}$ above the 97th centile in the curves according to Levene. ${ }^{10}$ CSF removal resulted in a significant increase in MFV and EDFV and a significant decrease in RI on each occasion when the ICP was $>7.0 \mathrm{~cm}$ $\mathrm{H}_{2} \mathrm{O}$. The CBFV was not influenced by CSF removal when the ICP was $<6.0 \mathrm{~cm} \mathrm{H}_{2} \mathrm{O}$. Therefore, we may conclude that an ICP of $6.0-7.0 \mathrm{~cm} \mathrm{H}_{2} \mathrm{O}$ is the upper limit of the normal ICP range in preterm infants with PHVD and that the ICP may be a better criterion for intervention than the Levene index alone.

The most important finding of our study is that, in $50 \%$ of the infants, removal of $5 \mathrm{ml} \mathrm{CSF} / \mathrm{kg} / \mathrm{tap}$ - that is, $10 \mathrm{ml} / \mathrm{kg} / 24$ $\mathrm{h}$ - did not result in normalisation of the ICP. In contrast with Volpe, ${ }^{22}$ we recommend not to start from a fixed tapping volume, but to take into account the ICP after tapping, and to drain as much and as frequently as necessary to normalise the ICP and to prevent swings in ICP.

From this study, it can be concluded that, in preterm infants with PHVD, repetitive CSF tapping from a ventricular access device guided by ICP measurements is a useful technique for determining the frequency and volume of CSF removal.

\section{Authors' affiliations}

W J Maertzdorf, A L M Mulder, C E Blanco, Department of Neonatology, University Hospital Maastricht, Maastricht, The Netherlands

J S H Vles, Department of Child Neurology, University Hospital Maastricht

E Beuls, Department of Neurosurgery, University Hospital Maastricht

\section{REFERENCES}

1 Claris O, Besnier S, Lapillonne A, et al. Incidence of ischemic-hemorrhagic cerebral lesions in premature infants of gestational age less than 28 weeks: a prospective ultrasound study. Biol Neonate 1996;70:29-34.
2 Roland EH, Hill A. Intraventricular hemorrhage and posthemorrhagic hydrocephalus. Clin Perinatol 1997;24:589-606.

3 Ventriculomegaly trial group. Randomised trial of early tapping in neonatal posthaemorrhagic ventricular dilatation. Arch Dis Child 1990:65:3-10.

4 du Plessis AJ. Posthemorrhagic hydrocephalus and brain injury in the preterm infant: dilemmas in diagnosis and management. Semin Pediatr Neurol 1998:5:161-79.

5 Volpe JJ. In: Volpe JJ, ed. Neurology of the newborn. 4th ed. Philadelphia: Saunders, 2001:428-93.

6 Punt J. Management of hydrocephalus in newborn infants. Semin Neonatol 1996;1:203-10.

7 Ventriculomegaly trial group. Randomised trial of early tapping in neonatal posthaemorrhagic ventricular dilatation: results at 30 months. Arch Dis Child Fetal Neonatal Ed 1994;70:F129-36.

8 Whitelaw A, Saliba E, Fellman V, et al. Phase I study of intraventricular recombinant tissue plasminogen activator for treatment of posthaemorrhagic hydrocephalus. Arch Dis Child Fetal Neonatal Ed 1996;75:F20-6.

9 Gavilanes AWD, Vles JSH, Beuls E, et al. Treatment of post-haemorrhagic hydrocephalus with a subcutaneous ventricular reservoir in preterm infants. Eur J Pediatr Neurol 1997;2/3A:849.

10 Levene MI. Measurement of the growth of the lateral ventricles in preterm infants with real-time ultrasound. Arch Dis Child 1981;56:900-4.

11 Pourcelot M. Application cliniques de l'examen Doppler transcutane. In: Peronneau, ed. Velocimetre ultrasonore Doppler. Paris: Inserm, 1974:213.

12 Levy ML, Masri LS, McComb JG. Outcome for preterm infants with germinal matrix hemorrhage and progressive hydrocephalus. Neurosurgery 1997;41:1111-17.

13 Haines SJ, Lapointe M. Fibrinolytic agents in the management of posthemorrhagic hydrocephalus in preterm infants: the evidence. Childs Nerv Syst 1999;15:226-34.

14 Gavilanes AWD, Heineman E, Herpers MHM, et al. Use of neonatal intensive care unit as a safe place for neonatal surgery. Arch Dis Child Fetal Neonatal Ed 1997;76:F51-3.

15 Mackamee LR, Gonzales Jl, Chance GW. Cerebral blood flow velocity profiles in intraventricular haemorrhage progressing to hydrocephalus [abstract]. Pediatr Res1998;43:224A.

16 Quinn MW, Ando Y, Levene MI. Cerebral arterial and venous flow-velocity measurements in post-haemorrhagic ventricular dilatation and hydrocephalus. Dev Med Child Neurol 1992;34:863-9.

17 Taylor G. Recent advances in neonatal cranial ultrasound and Doppler techniques. Clin Perinatol 1997;24:677-91.

18 Hanlo PW, Gooskens RH, Nijhuis IJ, et al. Value of transcranial Doppler indices in predicting raised ICP in infantile hydrocephalus. A study with review of the literature. Childs Nerv Syst 1995;11:595-603.

19 Bada HS. Intracranial monitoring: its role and application in neonatal intensive care. Clin Perinatol 1983:10:223-36.

20 Kaiser AM, Whitelaw AGL. Cerebrospinal fluid pressure during post haemorrhagic ventricular dilatation in newborn infants. Arch Dis Child 1985:60:920-4.

21 Kempley ST, Gamsu HR. Changes in cerebral artery blood flow velocity after intermittent cerebrospinal fluid drainage. Arch Dis Child 1993;69:74-6

22 Volpe JJ. In: Volpe LL, ed. Neurology of the newborn. 4th ed. Philadelphia: Saunders, 2000:428-96. 